\title{
POTENCIAL ALELOPÁTICO DIFERENCIAL DE CULTIVARES DE TRIGO (TRITICUM AESTIVUM L.) CHILENO SOBRE BALLICA ANUAL (LOLIUM RIGIDUM) VAR. WIMMERA
}

\author{
THE DIFFERENTIAL ALLELOPATHIC POTENTIAL OF CHILEAN WHEAT \\ CULTIVARS (TRITICUM AESTIVUM L.) ON ANNUAL RYEGRASS \\ (LOLIUM RIGIDUM) VAR. WIMMERA
}

Emma Bensch T. ${ }^{1}$; Heidi Schalchli S. ${ }^{1}$; Ricardo Fuentes $P^{2}{ }^{2}$ Peter Seemann $F^{2}$; Claudio Jobet $F^{3}$

\begin{abstract}
RESUMEN
Los objetivos de esta investigación fueron: validar el método ECAM (Wu et al., 2000a) y determinar el potencial alelopático de cincuenta cvs. de trigo cultivados en Chile sobre ballica anual (Lolium rigidum) var. Wimmera. El diseño experimental fue completamente al azar y en bloque con cuatro repeticiones. Se evaluó largo radical mayor de la especie receptora (ballica) a los diez días de establecida. Los datos transformados a porcentaje de inhibición del largo radical de la especie receptora en relación al testigo fueron sometidos a ANDEVA, Análisis Cluster y Duncan o Tukey $(\mathrm{P} \leq 0.05)$. El método ECAM modificado fue eficaz para evaluar el potencial alelopático de los cincuenta cultivares de trigo y, en general, se produjo inhibición del crecimiento radical de ballica con un rango de inhibición de 20 a 80\%. Los cultivares más alelopáticos fueron Tukan, Oracle y Castaño y los con menor potencial Baroudeur, Paillaco y Domo.
\end{abstract}

Palabras clave: Triticum aestivum, Lolium rigidum, cultivares, crecimiento radical, ECAM, alelopatía.

\begin{abstract}
The objectives of this investigation were: to validate the ECAM method and to validate the allelopatic potential of 50 wheat cultivars on Lolium rigidum var. Wimmera. The experimental design was completely at random or in blocks with four repetitions. The longest main root of the receptive species (lolium grass) was evaluated ten days after the establishment of the last one. The transformed data into percentage of inhibition of root length of the receptive species in relation to the control was analized using ANDEVA, cluster analysis, Duncan or Tukey (P $\leq 0.05)$. The modified ECAM method was efficient to evaluate the allelopathic potential of fifty wheat cultivars. In most cases root development of Lolium rigidum var. Wimmera was inhibited, the range of inhibition varied for lolium grass beteween 20 to $80 \%$. The most allelopathic cultivars were Tukan, Oracle and Castaño and with less potential Baroudeur, Paillaco and Domo.
\end{abstract}

Key words: Triticum aestivum, Lolium rigidum, cultivars, root length, ECAM, allelopathy.

\section{INTRODUCCIÓN}

La alelopatía ha sido utilizada en agroecosistemas con fines prácticos (Anaya, 1999; Anaya et al., 2001). Entre otros se cita la utilización de cultivos de supresión o cubiertas alelopáticas vegetales (Chou $e t$ al., 1987; Eskelsen y Crabtree, 1995; Chou, 1999; Ormeño, 1999), rotaciones alelopáticas o cultivos compañeros (Rice, 1979; Barnes y Putnam, 1986; Wojtkowiak et al., 1990; Pérez y Ormeño, 1991; Ormeño, 1992, 1999), incorporación de residuos de cultivos alelopáticos al suelo (Dilday et al., 1994; Chung y Miller, 1995; Ormeño, 1999) y uso de extractos fitotóxicos de plantas alelopáticas (Chung y Miller, 1995; Duke et al., 1997; Chou, 1999; Cheema y Khaliq, 2000). En el último tiempo, la

\footnotetext{
Universidad de La Frontera, Facultad de Ciencias Agropecuarias y Forestales.

2 Universidad Austral de Chile, Facultad de Ciencias Agrarias.

3 INIA, CRI Carillanca, Fitomejoramiento y Recursos Filogenéticos. E-mail: eabensch@ufro.cl
}

Fecha de Recepción: 25 Abril 2007

Fecha de Aceptación: 29 Mayo 2007 
investigación en el área se ha centrado en la búsqueda de germoplasma con potencial alelopático (Wu et al., 1999). En este sentido, la identificación de cultivares con alta actividad alelopática y la transferencia de esta característica a cultivares modernos podrían contribuir a restaurar una propiedad que inadvertidamente pudo haberse perdido durante el proceso de selección, cuando los cultivares se seleccionaron para otros fines.

Estudios en arroz (Dilday et al., 1994), avena (Fay y Duke, 1977), cebada (Ben-Hammouda y Oueslati, 1999), maravilla (Leather, 1983; Pérez, 1990), zapallo (Putnam y Duke, 1974; Lockerman y Putnam, 1979, citados por Wu et al., 1999) y trigo (Wu et al., 2001a) demuestran que hay variación en la actividad alelopática entre accesiones y que algunas de estas accesiones inhiben el desarrollo de otras especies vegetales.

Por la importancia del trigo a nivel mundial, se ha evaluado el potencial alelopático de accesiones de este cereal con resultados promisorios (Copaja et al., 1991; Bertholdsson, 2004; Wu et al., 1998, 1999, 2000a, 2000b, 2000c, 2001b; Zheng et al., 2007). En Chile no hay información publicada en el tema.

Por otra parte, los métodos de análisis son determinantes para evaluar inicialmente el potencial alelopático. Metodologías más comúnmente utilizadas en estudios alelopáticos son las descritas por Putman y Duke (1974), Fay y Duke (1977), Dilday et al. (1994) y Wu et al. (1998). En general, estos métodos requieren un extenso período de evaluación, difícilmente separan el componente alelopático del competitivo y pueden generar situaciones que alterarían la producción de aleloquímicos. Por estas razones es prioritario estandarizar la metodología. $\mathrm{Al}$ respecto, Wu et al. (2000a) proponen el Método de Análisis de Laboratorio ECAM (equal-compartment-agar-method), que permite simular la liberación natural de aleloquímicos desde plantas vivas donantes hacia un medio de cultivo estéril y sin nutrientes, evitando el efecto de microorganismos y la competencia por nutrientes, agua y luz entre las especies vegetales evaluadas.

En este contexto, como hipótesis de trabajo se postula que cultivares de trigo difieren en el efecto alelopático sobre ballica anual (Lolium rigidum var. Wimmera). Para dar cumplimiento a la hipótesis de trabajo se plantean como objetivos específicos: validar el método de análisis de laboratorio ECAM (Wu et al., 2000a) para las condiciones experi- mentales de la investigación, modificarlo si fuese necesario y evaluar cincuenta cultivares de trigo de Chile, en relación a su potencial alelopático diferencial sobre ballica anual (Lolium rigidum var. Wimmera).

\section{MATERIALES Y MÉTODOS}

La investigación se realizó en el Laboratorio de Cultivo de Tejidos Vegetales del Instituto de Protección y Sanidad Vegetal de la Facultad de Ciencias Agrarias de la Universidad Austral de Chile. Los cultivares de trigo (Triticum aestivum L.) de Chile fueron obtenidos del Banco de Germoplasma del Centro Regional de Investigación Carillanca, Temuco, dependiente del Instituto de Investigaciones Agropecuarias (INIA) y del predio experimental Maquehue, perteneciente a la Facultad de Ciencias Agropecuarias y Forestales de la Universidad de La Frontera, Temuco. Los cultivares de trigo de Australia provinieron de la Colección de Cereales de Invierno de Australia y fueron proporcionados por el Dr. G. Wu, Universidad Waga-Waga, Australia. Las semillas de ballica anual (Lolium rigidum var. Wimmera) se adquirieron en el comercio.

Para el establecimiento de los ensayos de laboratorio se utilizó el Método de Análisis de Laboratorio ECAM (Wu et al., 2000a), el cual fue modificado y ajustado para las condiciones de laboratorio donde se desarrolló la investigación.

Ajuste del método ECAM. Para adecuar el método a las condiciones de la investigación, se realizaron dos ensayos independientes y sucesivos, establecidos en un diseño completamente aleatorizado con tres repeticiones; el testigo fue la especie receptora Lolium rigidum var. Wimmera, descrita como especie sensible a los exudados radicales de trigo (Wu et al., 2000a, 2002). La evaluación del largo radical mayor de la especie receptora se realizó a los diez días de ubicada la semilla en el sistema. Se incluyeron nueve cultivares de trigo obtenidos de Chile (Agofen, Barros, Blanquillo, Cachiporra, Castaño Colorado, Colorado Torcaza, Copihue, Julio y Linaza) y uno de Australia (Triller), reportado como altamente alelopático (Wu et al., 2000a). Los cultivares de trigo identificados como más alelopáticos (Agofen, Blanquillo, Castaño Colorado y Triller, junto con Paleta y Pato) se evaluaron en un segundo ensayo, bajo similares condiciones y metodología. Los datos obtenidos fueron sometidos 
a Análisis de Varianza y comparación de medias por Prueba de Duncan $(\mathrm{P} \leq 0.05)$.

Para una mejor comprensión del método ECAM, propuesto por $\mathrm{Wu}$ et al. (2000a), se incluyen a continuación las etapas que éste considera.

a) Desinfección. Semillas de trigo (especie dadora de aleloquímicos) y ballica (especie receptora y sensible a aleloquímicos) son sumergidas en etanol (70\% por 2,5 min.), seguido de 4 enjuagues con agua destilada estéril; luego son sumergidas en hipoclorito de sodio (2,5\% por $15 \mathrm{~min}$.), seguido de 5 enjuagues con agua destilada estéril.

b) Pregerminación. Semillas de trigo y ballica (desinfectadas) son pregerminadas en cámara de cultivo iluminada a $25^{\circ} \mathrm{C}$, las semillas son dispuestas en placas de Petri con papel absorbente humedecido con agua destilada estéril como substrato. Las semillas de trigo son mantenidas en cámara de cultivo por 24 horas, luego enjuagadas con agua destilada estéril y vueltas a incubar por otras 24 horas; las de ballica son mantenidas en forma constante por 48 horas en cámara de cultivo.

c) Descripción general del método ECAM. En un frasco de vidrio con capacidad para $500 \mathrm{ml}$, que contiene un volumen de $30 \mathrm{ml}$ de medio de cultivo agar-agua al $3 \%$, sin nutrientes (esterilizado mediante autoclave a $120^{\circ} \mathrm{C}$ por $20 \mathrm{~min}$ ) se depositan 12 semillas pregerminadas con el embrión hacia arriba de cada cultivar de trigo en una de las mitades de la superficie del medio de cultivo, luego el sistema es cerrado con una lámina de parafilm (para evitar contaminación y evaporación desde la superficie del agar) y ubicado en cámara de cultivo por 7 días. Transcurrido este período, 12 semillas pregerminadas de ballica son seleccionadas y dispuestas en el compartimento no ocupado por el trigo; el sistema es separado verticalmente en dos compartimentos iguales por una lámina de papel blanco (previamente autoclavada), la que se sitúa a 1,0 cm sobre la superficie del medio de cultivo; nuevamente el sistema es cerrado con una lámina de parafilm y trasladado a cámara de cultivo por 10 días. Con el propósito de reducir la contaminación, la siembra de semillas debe realizarse bajo cámara de flujo laminar. A los 10 días postsiembra de la ballica se evalúa el largo radical mayor de esta especie. En este sistema los cultivares de trigo son considerados como tratamientos, en la confrontación trigo-ballica y el testigo corresponde a la relación ballica-ballica.

Potencial alelopático diferencial de cincuenta cultivares de trigo sobre $\boldsymbol{L}$. rigidum. Un total de cincuenta cultivares de trigo, cultivados o que se han dejado de cultivar en el sur de Chile, fueron evaluados en relación al efecto alelopático sobre ballica (Cuadro 1). Para la confrontación en el sistema se utilizó la relación de plántulas dadorreceptor de 12-12. La inhibición (\%) del largo radical se calculó en relación al testigo constituido por la confrontación ballica-ballica. Se utilizó el Método ECAM modificado, detallado en Resultados y Discusión.

Cuadro 1

Cultivares de trigo evaluados, ordenados según hábito de desarrollo

\begin{tabular}{|l|ll|ll|}
\hline \multicolumn{1}{|c|}{ Primaveral } & \multicolumn{2}{|c|}{ Invernal } & \multicolumn{2}{c|}{ Alternativo } \\
\hline Chifen & Renan & Tukan & Castaño & Quijote \\
Panquifen & Avital & Aztec & Huenufen & Renaico \\
Loncofen & Genial & Contra & Budifen & Dollinco \\
Toquifen & Baroudeur & Oracle & Rancofen & Bingo \\
Naofen & Forby & Oratorio & Lanco & Trauco \\
Carahue & Pepital & Orestis & Perquenco & \\
Dalcahue & Kona & Tilburi & Otto & \\
Malihue & Puken & Isangrein & Paleta & \\
Domo & Pankul & Tibet & Lumaco & \\
Antilhue & Texel & & Crac & \\
Fama & Rialto & & Metrenco & \\
Furio & Pajero & & & \\
\hline
\end{tabular}

Total cultivares: 12 primaverales, 21 invernales y 17 alternativos.

Fuente: Cartillas técnicas CRI Carillanca y Semillas Baer.

Comunicación personal: Claudio Jobet F. Ing. Agrónomo Ph.D, CRI Carillanca y

Juan Carlos García D. Ing. Agrónomo, Universidad de La Frontera. 
El diseño experimental fue de bloques completamente al azar con 4 repeticiones por tratamiento. Los cultivares de trigo fueron los tratamientos y el testigo fue un sistema ballica-ballica. Se consideraron 12 semillas de trigo y ballica por cada repetición. La evaluación del largo radical mayor de ballica se realizó 10 días postsiembra de esta especie. Los resultados fueron transformados a valores de porcentaje de inhibición del largo radical de la especie receptora en relación al testigo y sometidos a Análisis Cluster, para detectar diferencias entre los promedios de los grupos derivados del Análisis Cluster; se aplicó Prueba de Tukey $(\mathrm{P} \leq 0.05)$.

\section{RESULTADOS Y DISCUSIÓN}

Estudio preliminar y ajuste del Método ECAM. Se detectó efecto alelopático diferencial entre cultivares, en el caso del cultivar de Australia Triller, reportado como muy alelopático por Wu et al. (2000a); la inhibición del crecimiento radical sobre ballica fue superior comparado con los cultivares de Chile, de los cuales se desconocía su potencial alelopático, corroborando así su característica alelopática. Sólo el cv. Castaño colorado expresó similar comportamiento alelopático; en los otros cultivares la inhibición varió entre 25 y $50 \%$ respecto del testigo ballica-ballica. Este resultado fue coincidente con la caracterización alelopática reportada por los autores para el cv de Australia; asimismo, se generó información preliminar que permitió distinguir niveles extremos de alelopatía en los cvs. de trigo de Chile.

Se detectó que la habilitación del protocolo descrito por Wu et al. (2000a) precisaba de algunos ajustes para aminorar el nivel de contaminación de las plántulas y para habilitar la confrontación in vitro de la especie dadora (trigo) y receptora (ballica); además se observó que la intensidad lumínica de 3.560 lux fue excesiva, favoreciendo el desarrollo vegetativo por sobre el radical, lo que trajo consigo disminución de la fiabilidad de la evaluación. En este contexto se estimó desarrollar estudios tendientes a definir un protocolo, tanto de desinfección como de secuencia de acciones, que superara las restricciones anteriores y se adecuara a las condiciones de material biológico, equipo e infraestructura utilizados en esta investigación. Para superar la contaminación fúngica se evaluaron seis tratamientos. El mejor resultado se obtuvo con: in- mersión y agitación por 30 min de $100 \mathrm{~g}$ de semilla en solución acuosa de Benlate 50\% WP+Captan $80 \%$ WP $(1,8 \%+2,7 \%)$, lavado en agua destilada estéril durante cuatro veces sucesivas, inmersión en etanol (70\%) por 2,5 min, lavado durante cuatro veces en agua destilada estéril e inmersión final en hipoclorito de sodio $(2,5 \%)$ por $15 \mathrm{~min}$, lavado final en agua destilada estéril. Esta secuencia constituye una modificación al proceso de desinfección de la semilla descrito por Wu et al. (2000a).

Los ajustes para la habilitación del sistema ECAM fueron los siguientes: a) reemplazo de la lámina de papel que separa el frasco verticalmente por una lámina de cartulina firme de color blanco, a la que se le realizaron muescas en la parte superior para que quedase suspendida desde los bordes del frasco, sin tocar el medio de cultivo. Esta modificación evitó la absorción de agua desde el medio y el humedecimiento de la lámina de separación; b) disposición de la lámina de separación, antes de la siembra de ballica, para evitar que el follaje del trigo ocupe parte del espacio de la ballica; c) instalación de la lámina de cartón a $0,5 \mathrm{~cm}$ sobre la superficie del agar y no a $1,0 \mathrm{~cm}$, lo que restringió el crecimiento radical de trigo por sobre la superficie del agar hacia el lugar de la especie receptora, descartando el factor competitivo por espacio. El ajuste para pregerminado de la semilla consistió en disminuir a $24 \mathrm{~h}$ el período de pregerminación de ballica. Con esto se logró una mayor homogeneidad en la emergencia de esta especie y para trigo se mantuvo el período de $48 \mathrm{~h}$.

El protocolo ECAM modificado fue probado nuevamente para evaluar alelopatía sobre ballica de cinco cultivares de trigo de Chile (Blanquillo, Castaño colorado, Agofen, Paleta y Pato) y el cv. de Australia Triller, teniendo como testigo la relación ballica-ballica (Figura 1). Durante este experimento disminuyó la contaminación fúngica y hubo diferencia significativa entre los seis cultivares de trigo respecto del testigo, difiriendo significativamente de los otros cinco cultivares el cv. Blanquillo. Los valores de inhibición fluctuaron entre 41 y $79 \%$. Estos valores se enmarcan en los reportados por Wu et al. (2000a), quienes señalan cifras entre 24 y $90 \%$ de inhibición del largo radical de ballica por parte de líneas de trigos australianos, canadienses, chinos, sudafricanos y afganos. Con los resultados obtenidos se estima que el método ECAM modificado permitió evaluar eficazmente el proceso alelopático. Aislando la competencia por 


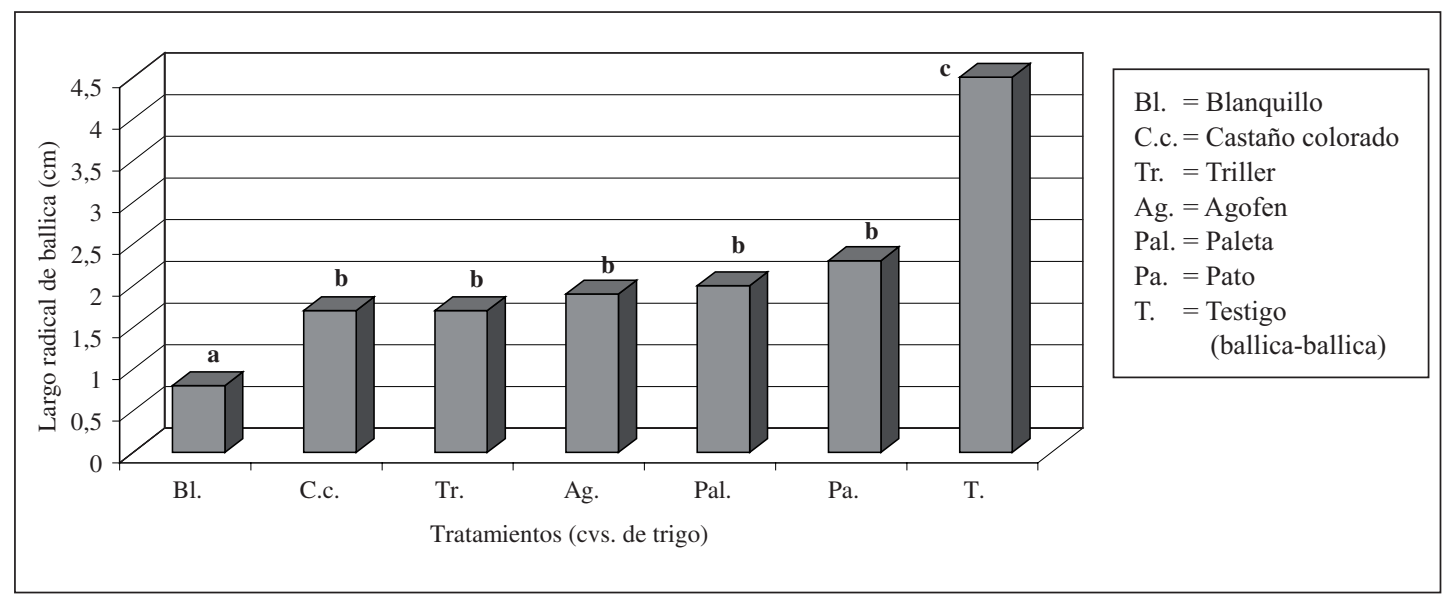

Figura 1. Efecto alelopático de seis cultivares de trigo sobre el largo radical de ballica.

espacio y luz disminuyó la contaminación fúngica y las raíces de las plantas donantes de trigo se distribuyeron regularmente en el medio de cultivo, interactuando adecuadamente con las de ballica en la relación dador-receptor.

En la Figura 2 se destaca la inhibición del sistema radical de la especie sensible (ballica) provocada por un cultivar de trigo con potencial alelopático negativo, en comparación al testigo.

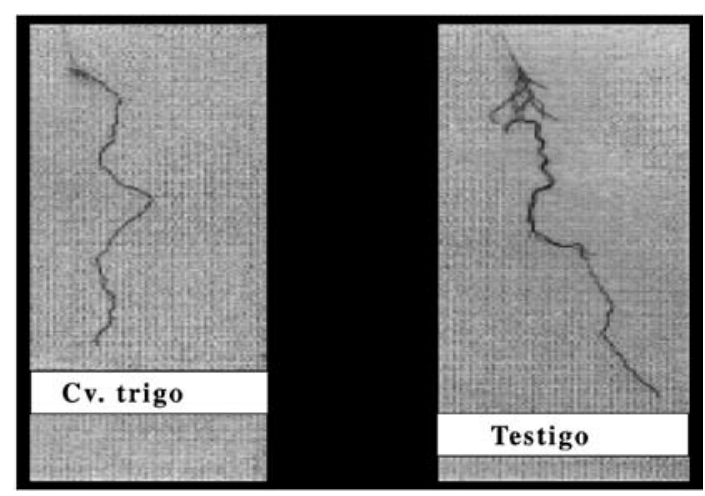

Figura 2. Inhibición del sistema radical de L. rigidum provocada por exudados radicales de cv. de trigo y del testigo (Lolium rigidum $\mathrm{L}$.$) .$

Potencial alelopático diferencial de cincuenta cultivares de trigo sobre $L$. rigidum. En el Cuadro 2 se incluye la inhibición del crecimiento radical de ballica para cada uno de los cultivares evaluados, los cuales variaron entre 20 y $80 \%$, con un promedio de $51,7 \%$ y un $50 \%$ de los cultivares por sobre este valor. Todos los cultivares ejercieron un efecto alelopático inhibitorio del largo radical con diferencia significativa entre los grupos; el promedio fue: Tukan ( $80 \%$ ), seguido de nueve cultivares con un promedio de $68,6 \%$, cinco con $65,0 \%$, seis con $59,8 \%$, tres con $53,3 \%$, once con $49,0 \%$, seis con $42,2 \%$, cinco con $35,6 \%$ y cuatro con $24,0 \%$. La menor inhibición se obtuvo con Baroudeur (20\%).

A partir del Análisis Cluster se constituyeron diez grupos principales, con un alto índice de similitud en el grupo y de disimilitud entre éstos, lo que indica que la acción alelopática de los cultivares que conformaron un mismo grupo es similar pero no idéntica; el promedio de estos grupos fue significativamente discriminado entre sí, en algunos casos un cultivar puede integrar dos grupos cuando el valor de inhibición está en el límite y se produce una interacción entre los grupos de disimilitud (Figura 3). Al comparar la disimilitud se estableció diferencia significativa (Figura 4 y Cuadro 3) con la significancia estadística obtenida; estos resultados demuestran la diversidad y distancia del potencial alelopático de los genotipos de trigo evaluados. Cabe hacer mención que los grupos quedaron conformados indistintamente por cultivares de distinto hábito, sin establecer tendencia de similitud en relación a este parámetro; el testigo conformó un grupo aparte.

Con los resultados obtenidos se corroboran los antecedentes reportados por Lemerle et al., 1996; Wu et al., 1998, 2000a y 2001b, en el sentido de que Lolium rigidum fue una especie altamente sensible a los exudados radicales de trigo y en consecuencia permitió discriminar con un alto grado de certeza entre los cultivares evaluados; tal así aconteció en 


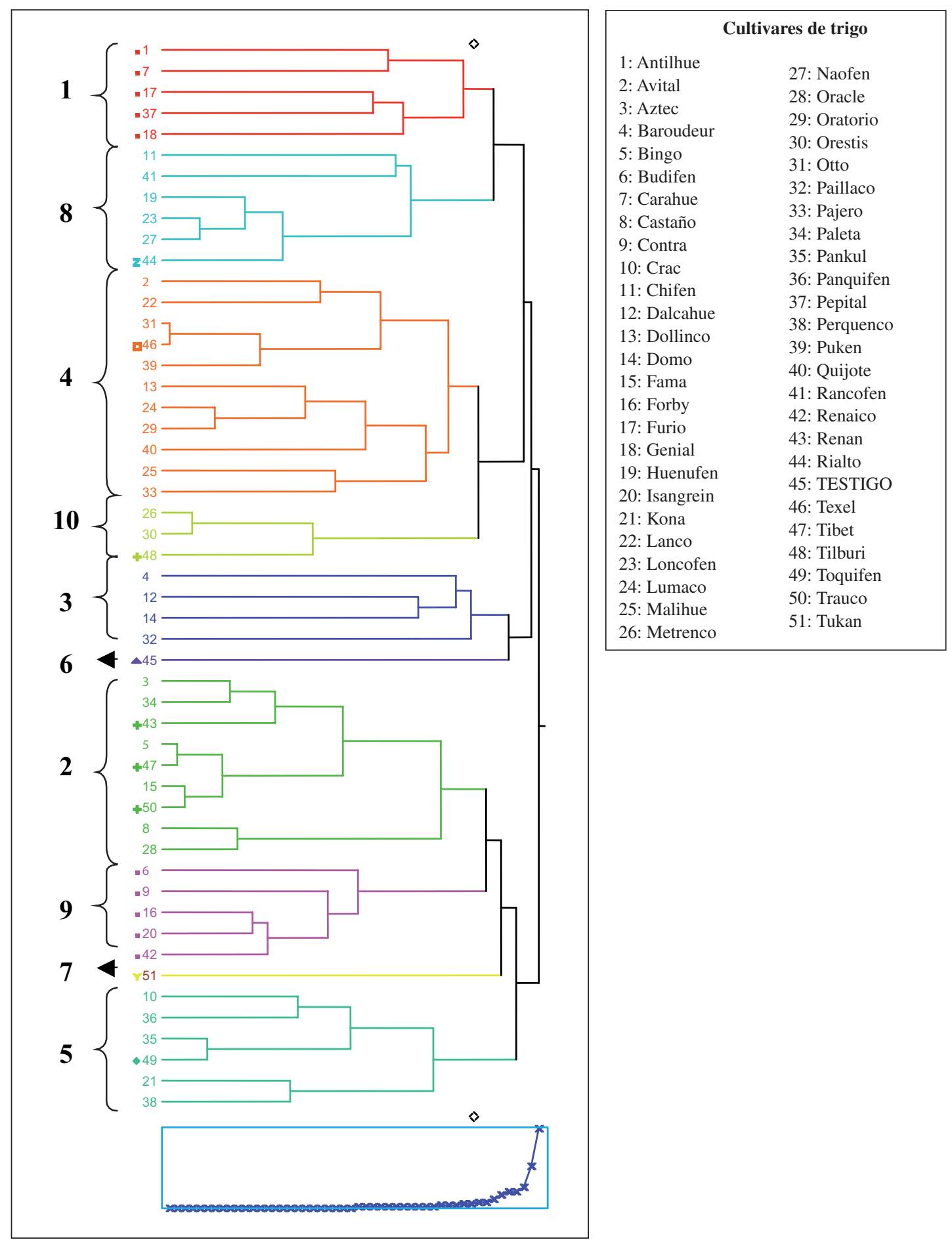

Figura 3. Dendrograma de similitud de cincuenta cultivares de trigo y el testigo en relación al potencial alelopático sobre L. rigidum. 


\section{Cuadro 2}

Inhibición (\%) del crecimiento radical de L. rigidum por exudados radicales de cultivares de trigo

\begin{tabular}{|l|c|}
\hline Cultivar & $\begin{array}{c}\text { Inhibición } \\
\text { Largo radical } \\
(\mathbf{\%})\end{array}$ \\
\hline Baroudeur & 20 \\
\hline Paillaco & 23 \\
\hline Domo & 24 \\
\hline Dalcahue & 29 \\
\hline Antilhue & 32 \\
\hline Carahue & 32 \\
\hline Pepital & 36 \\
\hline Furio & 39 \\
\hline Genial & 39 \\
\hline Rancofen & 39 \\
\hline Huenufen & 42 \\
\hline Naofen & 42 \\
\hline Loncofen & 43 \\
\hline Chifen & 43 \\
\hline Rialto & 44 \\
\hline Avital & 47 \\
\hline Texel & \begin{tabular}{c} 
(1) \\
\hline
\end{tabular} \\
\hline
\end{tabular}

\begin{tabular}{|l|c|}
\hline Cultivar & $\begin{array}{c}\text { Inhibición } \\
\text { Largo radical }^{(\mathbf{1})} \\
(\mathbf{\%})\end{array}$ \\
\hline Otto & 48 \\
\hline Quijote & 48 \\
\hline Puken & 49 \\
\hline Dollinco & 49 \\
\hline Lumaco & 49 \\
\hline Oratorio & 49 \\
\hline Lanco & 49 \\
\hline Malihue & 51 \\
\hline Pajero & 52 \\
\hline Tilburi & 52 \\
\hline Metrenco & 54 \\
\hline Orestis & 54 \\
\hline Perquenco & 57 \\
\hline Kona & 58 \\
\hline Crac & 60 \\
\hline Toquifen & 61 \\
\hline Panquifen & 61 \\
\hline
\end{tabular}

\begin{tabular}{|l|c|}
\hline Cultivar & $\begin{array}{c}\text { Inhibición } \\
\text { Largo radical }^{(\mathbf{1})} \\
(\boldsymbol{\%})\end{array}$ \\
\hline Pankul & 62 \\
\hline Contra & 63 \\
\hline Isangrein & 64 \\
\hline Renaico & 65 \\
\hline Forby & 66 \\
\hline Budifen & 67 \\
\hline Bingo & 67 \\
\hline Tibet & 67 \\
\hline Renan & 68 \\
\hline Fama & 68 \\
\hline Trauco & 68 \\
\hline Aztec & 69 \\
\hline Paleta & 69 \\
\hline Castaño & 70 \\
\hline Oracle & 71 \\
\hline Tukan & 80 \\
\hline
\end{tabular}

(1) Inhibición largo radical calculado en relación al testigo (ballica-ballica).

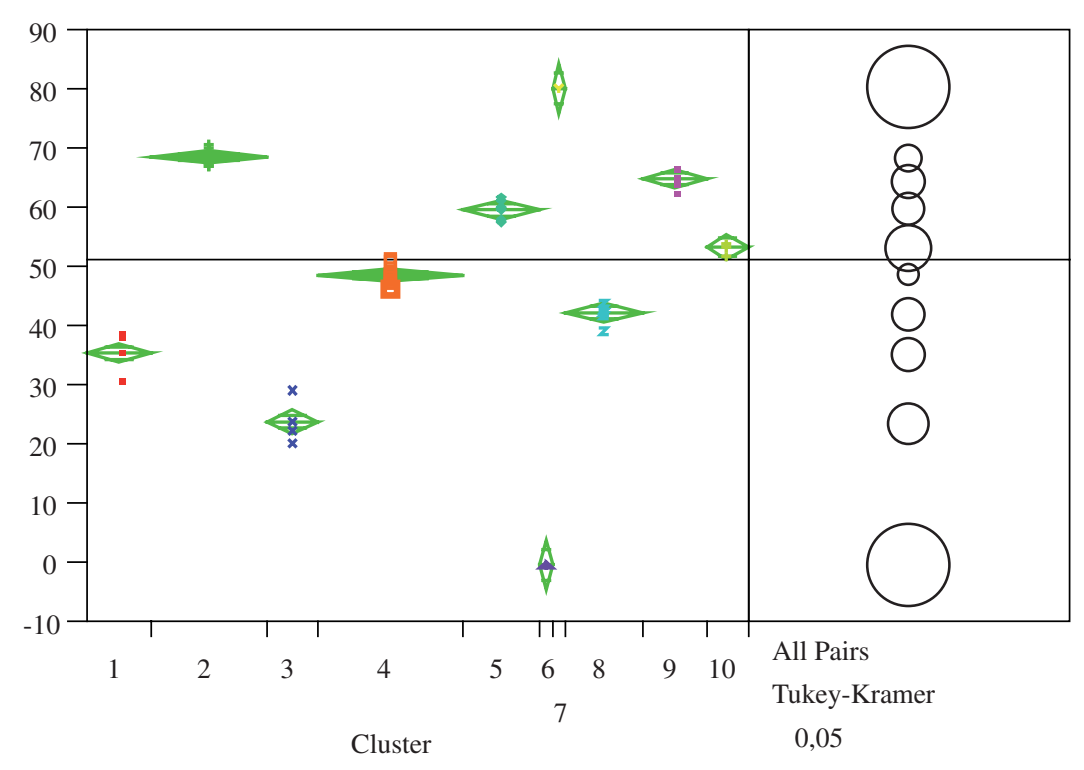

Figura 4. Gráfico de escala multidimensional y de distancia estadística para inhibición radical de L. rigidum. 


\section{Cuadro 3}

Significancia de disimilitud entre promedios de inhibición del largo radical (\%) de L. rigidum, para grupos Cluster de similitud conformados por cultivares de trigo evaluados

\begin{tabular}{|c|l|c|}
\hline $\begin{array}{c}\text { Grupos } \\
\text { Cluster }\end{array}$ & \multicolumn{1}{|c|}{ Cultivares que conforman cada grupo } & $\begin{array}{c}\text { Promedio } \\
\text { inhibición grupo } \\
(\%)\end{array}$ \\
\hline 1 & Antilhue, Carahue, Pepital, Furio, Genial & $35,6 \mathrm{~h}$ \\
\hline 2 & Bingo, Tibet, Fama, Trauco, Renan, Paleta, Aztec, Castaño, Oracle & $68,6 \mathrm{~b}$ \\
\hline 3 & Baroudeur, Paillaco, Domo, Dalcahue & $24,0 \mathrm{i}$ \\
\hline 4 & Avital, Otto, Texel, Quijote, Lanco, Puken, Dollinco, Lumaco, Oratorio, Malihue, Pajero & $49,0 \mathrm{f}$ \\
\hline 5 & Perquenco, Kona, Crac, Panquifen, Toquifen, Pankul & $59,8 \mathrm{~d}$ \\
\hline 7 & Tukan & $80,0 \mathrm{a}$ \\
\hline 8 & Rancofen, Huenufen, Naofen, Loncofen, Chifen, Rialto & $42,2 \mathrm{~g}$ \\
\hline 9 & Contra, Isangrein, Renaico, Forby, Budifen & $65,0 \mathrm{c}$ \\
\hline 10 & Tilburi, Orestis, Metrenco & $53,3 \mathrm{e}$ \\
\hline
\end{tabular}

La inhibición del largo radical se calculó en relación al testigo (ballica-ballica).

L. rigidum integró el grupo 6 del Análisis Cluster.

Letras distintas entre grupos indican diferencia significativa según Tukey $(\mathrm{P} \leq 0,05)$.

esta investigación. Otro aspecto que se reporta dice relación con la estimulación del crecimiento radical de ballica mediado por los exudados radicales de trigo (Wu et al., 2001b), efecto no observado en este estudio.

\section{CONCLUSIONES}

1. El método ECAM modificado permitió evaluar eficazmente el proceso alelopático entre la especie dadora (Triticum aestivum) y la especie receptora (ballica anual).

\section{LITERATURA CITADA}

ANAYA, A. 1999. Allelopathy as tool in the management of biotic resources in Agroecosystems. Critical Reviews in Plant Sciences 18:697-739.

ANAYA, A.; CRUZ-ORTEGA, R. 2001. La alelopatía: algunos estudios de caso y posibles aplicaciones. In: Anaya, A., Espinosa-García, F. y Cruz-Ortega, R. (eds). Relaciones químicas entre organismos: aspectos básicos y perspectivas de su aplicación. Instituto de Ecología, UNAM. Plaza y Valdés, S.A. de C.V. México. pp. 33-68.

BARNES, J.; PUTNAM, A. 1986. Evidence for allelopathy by residues and aquous extract of rye (Secale cereale L.). Weed Science 34:384-390.

BEN-HAMMOUDA, M.; OUESLATI, O. 1999. A germination bioassay to test the allelopathic potential of barley. RACHIS Newsletter 18:51-54.
2. Los cincuenta cultivares de trigo ejercieron efecto alelopático inhibitorio significativo sobre el crecimiento radical de Lolium rigidum var. Wimmera; el rango varió entre 80 y $20 \%$, con $50 \%$ de los cultivares por sobre el $50 \%$ de inhibición.

3. Los cultivares con mayor potencial alelopático inhibitorio (rango: 70-80\%) fueron Tukan, Oracle y Castaño y los con menor potencial alelopático inhibitorio (rango: 20-24\%) Baroudeur, Paillaco y Domo.

BERTHOLDSSON, N. 2004. Variation in allelopathic activity in spring wheat. In: Proceedings of Second European Allelopathy Symposium-Allelopathic: from understanding to application. p. 22. Pulawy, Poland, June 4, 2004.

CHEEMA, Z.; KHALIQ, A. 2000. Use of sorghum allelopathic properties to control weeds in irrigated wheat in a semi arid region of Punjab. Agriculture, Ecosystems and Environment 79:105-112.

CHOU, CH.; HWANG, S.; PENG, C.; WANG, Y.; HSU, F.; CHUNG, N. 1987. The selective allelopathic interaction of pasture-forest intercropping in Taiwan. Plant Soil 98:31-41.

CHOU, CH. 1999. Roles of allelopathy in plant biodiversity and sustainable agriculture. Critical Reviews in Plant Sciences 18:609-636. 
CHUNG, I.; MILLER, D. 1995. Natural herbicide potential of alfalfa residue on selected weed species. Agronomy Journal 87:920-925.

COPAJA, S.; NIEMEYER, H.; WRATTEN, S. 1991. Hydroxamic acids levels in Chilean and British wheat seedlings. Annals of Applied Biology 118: 223-227.

DILDAY, S.; LIN, J.; YANG, W. 1994. Identification of allelopathy in the USDA-ARS rice germplasm collection. Australian Journal of Experimental Agriculture 34:907-910.

DUKE, S.; SMEDA, R.; WESTON, L. 1997. Potential for utilization of allelopathy for weed management. In: Palestras e mesas redondas. XXI Congresso Brasileiro da cîencia das plantas daninhas. 6 a 11 de julho de 1997.

ESKELSEN, S.; CRABTREE, G. 1995. The role of allelopathy in Buckwheat (Fagorypum sagittatum) inhibition of Canada thistle (Cirsum arvense). Weed Science 43:70-74.

FAY, P.; DUKE, W. 1977. An assessment of allelopathic potential in Avena germplasm. Weed Science 25:224-228.

LEATHER, G. 1983. Sunflowers (Helianthus annus) are allelopathic to weeds. Weed Science 31:37-42.

LEMERLE, D.; VERBEEK, B.; COUSENS, R.; COOMBES, N. 1996. The potential for selecting wheat varieties strongly competitive against weeds. Weed Research 36:505-513.

ORMEÑO, J. 1992. Efectos alelopáticos del centeno (Secale cereale $\mathrm{L}$.) sobre malezas que crecen normalmente asociadas a cereales. XI Congreso de la Asociación Latinoamericana de Malezas (ALAM). Viña del Mar. 23-27 de noviembre.

ORMEÑO, J. 1999. Manejo y control de malezas con plantas alelopáticas: Centeno. In: Agricultura orgánica. Céspedes, C. y Carvajal, P. Trama. Talcahuano. pp. 121-137.

PÉREZ, F. 1990. Allelopathic effect of hydroxamic acids from cereals on Avena fatua L. and A. sativa L. Phytochemistry 29:773-776.

PÉREZ, F.; ORMEÑO, J. 1991. Difference in hidroxamic acid in roots and roots exudates of wheat (Triticum aestivum L.) and rye (Secale cereale L.) possible role in allelopathy. Journal of Chemical Ecology 17:1037-1043.

PUTNAM, A.; DUKE, V. 1974. Biological suppression of weeds: evidence for allelopathy in accessions of cucumber. Science 185:370-372.
RICE, E. 1979. Allelopathy. New York. Orlando, Academy press. $353 \mathrm{p}$.

WOJTKOWIAK, D., POLITYCKA, B., SCHNEIDER, M.; PERKOWSKI, J. 1990. Phenolic substances as allelopathic agents aresing during the degradation of rye (Secale cereale L.) tissues. Plant Soil 124:143-147.

WU, H.; HAIG, T.; PRATLEY, J.; LEMERLE, D. $2000 \mathrm{~b}$. Distribution and exudation of allelochemicals in wheat Triticum aestivum. Journal of Chemical Ecology 26:2141-2154.

WU, H., HAIG, T., PRATLEY, J., LEMERLE, D.; AN M. 2002. Biochemical basis for wheat seedling allelopathy on the suppression of annual ryegrass (Lolium rigidum). Journal of Agricultural and Food Chemistry 50:4567-4571.

WU, H.; PRATLEY, J.; LEMERLE, D.; HAIG, T. 1999. Crop cultivars with allelopathic capability. Weed Research 39:171-180

WU, H.; PRATLEY, J.; LEMERLE, D.; HAIG, T. 2000a. Laboratory screening for allelopathic potential of wheat (Triticum aestivum) accessions against annual ryegrass (Lolium rigidum). Australian Journal Research 51:259-266.

WU, H.; PRATLEY, J.; LEMERLE, D.; HAIG, T. 2001 . Allelopathy in wheat (Triticum aestivum). Annals of Applied Biology 139:1-9.

WU, H.; PRATLEY, J.; LEMERLE, D.; HAIG, T. 2001b. Wheat allelopathic potential against a herbicide-resistant biotype of annual ryegrass. In: Proceeding of the $10^{\text {th }}$ Australian Agronomy Conference. Hobart, Australia.

WU, H.; PRATLEY, J.; LEMERLE, D.; HAIG, T.; VERBEEK, B. 1998. Differential allelopathic potential among wheat accessions to annual ryegrass. $I n$ : Proceeding of the $9^{\text {th }}$ Australian Agronomy Conference (Eds. D.L. Michalk, J.E. Pratley). Wagga-Wagga, NSW. pp. 567-571.

WU, H.; PRATLEY, J.; LEMERLE, D.; HAIG, T. 2000c. Evaluation of seedling allelopathy in 453 wheat (Triticum aestivum) accessions against annual ryegrass (Lolium rigidum) by the equal-compartment-agar-method. Australian Journal of Agricultural Research. 51: 937-944.

ZHENG, Y.; ZHAO, Y: DONG, F.; LIU, J.; YAO, J.; HURLE, K. 2007. Allelopathic effects of wheat extracts and DIMBOA on weeds. Allelopathy Journal 19: 171-178. 